\title{
Madres niñas-adolescentes de 14 años y menos. Un grave problema de salud pública no resuelto en Chile
}

\author{
Ramiro Molina C, Temístocles Molina Ga, \\ Electra González $\mathrm{A}^{\mathrm{b}}$.
}

\section{Teenage fecundity rates in Chile. A serious public health problem}

Background: Teenage fecundity rates are an indicator of epidemiological discrimination in developing countries. Aim: To study fertility rates of girls under 14 years of age in Chile from 1993 to 2003. Material and methods: Information of children born alive from mothers aged 10 to 15 years, was obtained from the Chilean National Institute of Statistics. Age segmented population data was obtained from the Ministry of Health. Trends were analyzed by regions and single ages. The rates in communities of the Metropolitan Region were compared. Results: Between 1993 and 2003, there was an increasing trend in fecundity rates, ratios and crude numbers. These rates duplicate from 14 to 15 years of age. In the Metropolitan Region, the fecundity ratios of communities with lower economical incomes is seven times greater than those with higher incomes. During 2003, the fecundity rates in Chile were 100 and 10 higher than those of Holland and Sweden in 1981. Conclusions: In developing countries with very low infant mortality rates such as Chile, the high fecundity rates of young girls is an indicator of a deficient human and social development. Sexual Education and Health Services for adolescents are essential to prevent this public health problem (Rev Méd Chile 2007; 135: 79-86).

(Key words:

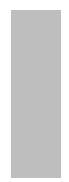

Recibido el 12 de diciembre, 2005. Aceptado el 7 de agosto, 2006.

Centro de Medicina Reproductiva y Desarrollo Integral de la Adolescencia (Cemera), Facultad de Medicina, Universidad de Chile.

aEstadístico, Prof. Asistente

${ }^{\mathrm{b}}$ Asistente Social, Prof. Asistente

T a mortalidad infantil ha sido un indicador del

nivel de salud, bienestar y desarrollo social de

Correspondencia a: Dr. Ramiro Molina C. Centro de Medicina Reproductiva y Desarrollo Integral de la Adolescencia (Cemera), Facultad de Medicina, Universidad de Chile. Fax: 56-2-7356518. E mail: cemera@med.uchile.cl una población 1 . Cuando baja a menos de dos dígitos, no se diferencia por clase social. En esta situación, la fecundidad adolescente es un indicador de mayor discriminación epidemiológica. La mortalidad infantil en países como Costa Rica, Cuba, Chile y Uruguay, alrededor de 2004, era menor de 9 por 1.000 nacidos vivos (NV) y en 
países como Canadá, Estados Unidos, Finlandia, Suecia, Alemania y Francia estaba entre 3 y 7 por $1.000 \mathrm{NV}^{2}$. En tanto que la proporción de madres de 19 años y menos era alrededor de $16 \%$ a $18 \%$ en los países en desarrollo antes mencionados y $3 \%$ en los países desarrollados, con excepción de Estados Unidos que llegaba a $9 \%{ }^{3}$.

La fecundidad adolescente debe ser examinada en asociación con otros indicadores económicos y sociales, para medir el impacto de las medidas en salud, educación y de intervención social en la adolescencia y juventud. En Chile, se ha medido la asociación entre la maternidad adolescente y el mayor riesgo de mobimortalidad materna y perinatal, en comparación con madres mayores de 24 años ${ }^{4}$.

Se define como población adolescente a la comprendida entre 10 y 19 años y tiene tres etapas: temprana, entre los 10 y 13, media entre 14 y 16, y tardía entre los 17 y 19 años ${ }^{5}$. La fecundidad se expresa de 15 a 49 años o mujeres en edad de procrear y no incluye a la adolescencia temprana de 14 años y menos y parte de la adolescencia media ${ }^{6}$. Esto ocurre por la dificultad para estimar la fertilidad por debajo de los 12 años $\mathrm{y}$, por ende, las tasas de fecundidad. La menarquia marca el límite del inicio de la fecundidad y su edad promedio en Chile se estima en 12,2 años. Otro factor que afecta la estimación de la fertilidad en este período, es la alta proporción de ciclos anovulatorios entre los 12 y 14 años que alcanza entre $55 \%$ y $82 \%{ }^{7,8}$. En estas circunstancias no es factible hablar de fecundidad en las menores de 15 años, pues el denominador está afecto a un error natural. La maternidad en estas edades, además del riesgo para la salud de la adolescente, si el embarazo no tiene un control prenatal preferencial, implica además la eventual existencia de abuso sexual.

La maternidad adolescente es un problema emergente en salud pública que cruza a sectores como educación, salud, justicia, derechos del niño y de la mujer, por lo cual es considerado como un indicador de desarrollo y bienestar social. El Ministerio de Salud ha propuesto para 2010, la meta de disminuir a cero los embarazos en las mujeres de 14 años y menos ${ }^{9}$.

Este estudio describe la información de fecundidad en adolescentes tempranas con el fin de estimular su estudio en mayor profundidad, por otros investigadores, organismos del estado en Chile y otros países de la región.

\section{MÉTodo}

Es un estudio descriptivo que aplica indicadores clásicos de fecundidad adolescente temprana y otros adaptados para observar la importancia del fenómeno según nivel socioeconómico en la Región Metropolitana.

La información de NV por regiones y por edades simples de madres de 10 a 15 años, en los últimos 11 años y la población de estas edades, se obtuvo en el Instituto Nacional de Estadísticas y en el Ministerio de Salud, respectivamente. La expresión de la fecundidad en menores de 14 años se denomina "razón", pues se incluye en el denominador al total de personas entre 11 y 14 años y no todas están en edad fértil. Se prefirió usar el mismo criterio para todos los cálculos y no estimar por los dos factores: edad de la menarquia y proporción de anovulación. La información se analiza en cifras crudas, proporciones, tasas y razones. A nivel comunal, no fue posible depurar el grupo de 10 años y se utilizó la población de 11 a 14 años. No se aplicaron pruebas de significación en las tasas y razones, por ser datos continuos y tampoco en la comparación de comunas, por ser muy evidentes las diferencias en los extremos socioeconómicos y estar afectos a variables que este estudio no puede controlar.

Se utilizó la información de las raciones alimenticias que el Ministerio de Educación entrega por comuna, como indicador socioeconómico, dado que aumentan proporcionalmente a medida que los escolares tienen mayores necesidades. Otros indicadores no son tan específicos de la población en edad escolar, donde se concentra este estudio. La raciones se expresan como proporción del total de población por comuna ${ }^{10}$.

En la serie analizada, se encontró una sola madre de 10 años, correspondiente a la región de los Lagos en 2001. Así, se incluyó a la población de 11 años y más.

\section{Resultados}

La Tabla 1 muestra que la Región Metropolitana acumula un tercio de los NV del país, otro 38\% 
Tabla 1. Nacidos vivos de madres adolescentes de 11 a 14 años por regiones seleccionadas y de 15 años de edad en el total del país. C hile 1993-2003

\begin{tabular}{|lrrrrrrrrrr|}
\hline Año/R & V & VII & VIII & X & Met & Otras* & Total & $\begin{array}{r}\text { Razón x } \\
10 \text { mil }\end{array}$ & 15 años & $\begin{array}{r}\text { Tasa x } \\
1.000\end{array}$ \\
\hline 1993 & & & & & & & & & & \\
1994 & 79 & 89 & 104 & 86 & 233 & 221 & 863 & 17,6 & 2.118 & 17,8 \\
1995 & 67 & 78 & 106 & 88 & 289 & 251 & 879 & 17,5 & 2.293 & 19,3 \\
1996 & 101 & 82 & 118 & 83 & 338 & 311 & 1.033 & 20,1 & 2.511 & 21,1 \\
1997 & 106 & 92 & 135 & 107 & 374 & 312 & 1.126 & 21,4 & 2.770 & 22,6 \\
1998 & 97 & 76 & 143 & 119 & 439 & 309 & 1.183 & 22,0 & 3.106 & 24,7 \\
1999 & 102 & 92 & 134 & 101 & 439 & 277 & 1.175 & 21,4 & 2.884 & 22,3 \\
2000 & 78 & 73 & 131 & 98 & 362 & 310 & 1.052 & 18,8 & 2.704 & 20,3 \\
2001 & 104 & 74 & 105 & 94 & 374 & 304 & 1.055 & 18,4 & 2.763 & 20,2 \\
2002 & 115 & 92 & 132 & 103 & 397 & 322 & 1.161 & 20,2 & 2.827 & 20,4 \\
2003 & 106 & 87 & 132 & 101 & 378 & 314 & 1.118 & 19,3 & 2.604 & 18,5 \\
Total & 99 & 63 & 125 & 93 & 343 & 271 & 994 & 17,1 & 2.414 & 16,9 \\
Promedio & 1.054 & 898 & 1.365 & 1.073 & 3.966 & 3.283 & 11.639 & 19,4 & 28.994 & 20,3 \\
$\%$ & 95,8 & 81,6 & 124,1 & 97,5 & 360,5 & 298,5 & $1.058,1$ & & $2.635,8$ & \\
Razón x 10.000 & 9,1 & 7,7 & 11,7 & 9,2 & 34,1 & 28,2 & 100,0 & & & \\
\hline
\end{tabular}

*Corresponde a 8 regiones del país: I-II-III-IV-VI-IX-XI y XII.

lo aportan las 4 regiones con alta fecundidad adolescente y el $28 \%$ restante se distribuye en las otras 8 regiones. En el total, el promedio de NV de madres de 11 a 14 años, alcanzó a 1.058. La razón por 10.000 mujeres en esa edad aumentó hasta 2002, registrándose una razón más baja en 2003 en todas las regiones. Los aumentos de las cifras crudas se observaron en todas las regiones.

La razón por 10.000 mujeres de esa edad fue más alta en las regiones VII y X con 4 y 5 puntos por encima del promedio del total del país $(19,4)$. Las niñas-madres de 15 años aumentaron a casi 29.000, es decir, a esta edad aumentaron a más del doble y se expresan como tasa específica de fecundidad en esa edad. Esta expresión muestra que el riesgo de embarazo del grupo de 11 a 14 años aumentó más de 10 veces al cumplir los 15 años, de 19,4 a 203 por 10.000 mujeres de esa edad.

En la penúltima fila de la Tabla 2, se observa que el promedio de NV en la serie, aumentó 12 veces entre las mujeres de 11 años y aquellas de
12 años, 9 veces más en la edad de 12 a 13; 5 veces más en la edad de 13 a 14 años y 3 veces entre los 14 y 15 años. El 98\% de los NV de las menores de 14, era aportado por las mujeres de 13 y 14 años. La razón de fecundidad (última fila), sigue la misma tendencia.

Hay un aumento progresivo de las cifras crudas de NV en las mujeres de 13 y 14 años con variabilidad de un año a otro en las de 12 años. Esto se refleja en las cifras y razones que aumentan en las adolescentes de 11 a 14 y de 11 a 15 años con excepción de 2003. El contingente de 15 años mantuvo la proporción de $70 \%$ a $72 \%$ del total de NV en el grupo de 11 a 15 años (recíproca de la novena columna). El promedio anual de 3.700 nuevas madres entre 11 y 15 años es definitivamente un problema emergente de salud pública. Se ha incluido la información de la fecundidad entre 15 y 19 años, que muestra una tendencia al descenso, y es la información que regularmente se publica, dando la falsa imagen de descenso de la fecundidad adolescente. 
Tabla 2. Fecundidad de 11 a 15 años por edades simples en comparación con la fecundidad de grupos de adolescentes de mayor edad. C hile 1993-2003

\begin{tabular}{|c|c|c|c|c|c|c|c|c|c|c|c|c|}
\hline Año & 11 & 12 & 13 & 14 & 15 & $\begin{array}{c}\text { Subtotal } \\
11-14\end{array}$ & $\begin{array}{l}\text { Subtotal } \\
11-15\end{array}$ & $\begin{array}{c}\% \\
11-14 \mathrm{a} \\
11-15 \mathrm{a}\end{array}$ & $\begin{array}{l}\text { Razón* } \\
11-15 \text { a } \\
\text { (x 1.000) }\end{array}$ & $\begin{array}{l}\mathrm{NV} \\
16-19\end{array}$ & $\begin{array}{l}\text { NV 15-19 } \\
\text { y total } \\
11-19 \text { años }\end{array}$ & $\begin{array}{c}\text { Tasa** } \\
\text { 15-19 años } \\
\text { (x 1.000) }\end{array}$ \\
\hline 1993 & 1 & 18 & 144 & 700 & 2.118 & 863 & 2.981 & 29,0 & 4,9 & 34.582 & 37.563 & 63,6 \\
\hline 1994 & 0 & 14 & 148 & 717 & 2.293 & 879 & 3.172 & 28,3 & 5,1 & 34.797 & 37.969 & 65,1 \\
\hline 1995 & 3 & 21 & 182 & 827 & 2.511 & 1.033 & 3.544 & 29,1 & 5,6 & 34.308 & 37.852 & 65,6 \\
\hline 1996 & 3 & 23 & 184 & 916 & 2.770 & 1.126 & 3.896 & 28,9 & 6,0 & 34.679 & 38.575 & 65,2 \\
\hline 1997 & 1 & 20 & 185 & 977 & 3.106 & 1.183 & 4.289 & 27,6 & 6,5 & 35.262 & 39.551 & 65,2 \\
\hline 1998 & 3 & 25 & 207 & 940 & 2.884 & 1.175 & 4.059 & 28,9 & 6,0 & 36.296 & 40.355 & 65,0 \\
\hline 1999 & 1 & 18 & 172 & 861 & 2.704 & 1.052 & 3.756 & 28,0 & 5,4 & 35.387 & 39.387 & 61,9 \\
\hline 2000 & 2 & 20 & 183 & 850 & 2.763 & 1.055 & 3.818 & 27,6 & 5,4 & 35.439 & 39.257 & 60,3 \\
\hline 2001 & 3 & 29 & 199 & 930 & 2.827 & 1.161 & 3.988 & 29,1 & 5,6 & 34.734 & 38.722 & 58,3 \\
\hline 2002 & 0 & 18 & 194 & 906 & 2.604 & 1.118 & 3.722 & 30,0 & 5,2 & 32.778 & 36.500 & 53,8 \\
\hline 2003 & 0 & 12 & 155 & 827 & 2.414 & 994 & 3.408 & 29,2 & 4,7 & 30.430 & 33.838 & 48,9 \\
\hline Total & 17 & 218 & 1.953 & 9.451 & 28.994 & 11.639 & 40.633 & 28,6 & 5,5 & 378.936 & $\begin{array}{c}419.569+ \\
11.639 \\
431.208\end{array}$ & 60,9 \\
\hline$\% 11-14$ & 0,1 & 1,9 & 16,8 & 81,2 & - & $100,0^{\circ}$ & & & & & $2,7 \%$ & \\
\hline$\% 11-15$ & 0,04 & 0,54 & 4,8 & 23,3 & 71,4 & - & 100,0 & & & & $9,4 \%$ & \\
\hline $\begin{array}{l}\% 11-19 \\
\text { Promedio }\end{array}$ & 0,003 & 0,05 & 0,45 & 2,2 & 6,7 & -- & - & - & -- & 87,9 & $100,0^{* * 1 *}$ & \\
\hline $\begin{array}{l}\text { anual } \\
\text { Razón x }\end{array}$ & 2,0 & 2,0 & 178 & 859 & 2.636 & 1.058 & $3.694,0$ & -- & -- & 34.449 & 38.143 & \\
\hline 10.000 & 0,1 & 1,4 & 13,1 & 64,9 & 203,2 & 19,4 & 547,2 & - & - & & & \\
\hline
\end{tabular}

* Se expresa con el término razón por incluir a población que aún no ha tenido la menarquia, como denominador y por lo tanto sin posibilidades de ser fértil. Se usa por 1.000 para más fácil comparación con la 15 a 19 años de edad (última columna).

* Se expresa como tasa por incluir en el denominador a población en posibilidades (riesgo epidemiológico) de ser fértil.

*** Se expresa el grupo 11 a 19 años para el cálculo de la importancia relativa por edad. En las estadísticas vitales sanitarias, no se incluye los embarazos entre los 11 y 14 años.

La Tabla 3 selecciona algunas comunas de la Región Metropolitana ordenadas por la razón de fecundidad de 14 años y menos. Se incluyó como referencia al grupo de 15 a 19 años, que tiene un comportamiento semejante, sólo en las comunas de los extremos superior e inferior de la tabla. Se observa mayor proporción de raciones alimenticias anuales entregadas a escolares de las comunas de Cerrillos, La Pintana, Cerro Navia, Lo Prado, La Granja, El Bosque y San Ramón. Las comunas que recibieron menor proporción de raciones alimenticias para escolares fueron $\mathrm{La}$ Reina, Las Condes, Providencia y Vitacura. Esto revela indirectamente las necesidades alimenticias de los escolares de estas comunas y que se relaciona con el nivel socioeconómico de su población.

Por otra parte, la fecundidad fue más alta en las comunas que reciben mayor proporción de raciones alimenticias escolares y fue más baja en 
Tabla 3. $\mathrm{N}$ acidos vivos en adolescentes de 14 años y menos en comunas seleccionadas de la Región M etropolitana 2002 y raciones alimenticias escolares anuales 2004. Razón por 10.000 adolescentes de 10-14 años y tasa por 1.000 adolescentes de 15 -19 años

\begin{tabular}{|c|c|c|c|c|c|c|c|c|}
\hline \multirow[t]{2}{*}{ Comuna } & \multicolumn{2}{|c|}{$\begin{array}{l}\text { № de N.V. por } \\
\text { edad de la madre }\end{array}$} & \multicolumn{2}{|c|}{$\begin{array}{l}\text { Fecundidad } 14 \text { y (-) } \\
\text { años (x 10.000)*** }\end{array}$} & \multicolumn{2}{|c|}{$\begin{array}{l}\text { Fecundidad 15-19 } \\
\text { años (x 1.000) }\end{array}$} & \multirow{2}{*}{\multicolumn{2}{|c|}{$\begin{array}{c}\% \mathrm{de} \\
\text { Raciones/d/ } \\
\text { x Pob.Tot(*) } \\
\%\end{array}$}} \\
\hline & 14 y (-) & $15-19$ & Población & Tasa/Pror & Población & Tasa/Prom & & \\
\hline Cerrillos & 11 & 171 & 3.093 & 35,6 & 2.967 & 57,6 & & 9,3 \\
\hline La Pintana & 28 & 805 & 9.866 & 28,3 & 9.212 & 87,3 & & 10,0 \\
\hline Cerro Navia & 13 & 422 & 6.643 & 19,6 & 6.162 & 68,5 & & 7,6 \\
\hline Lo Prado & 8 & 236 & 4.265 & 18,8 & 4.150 & 56,9 & 71,2 & 6,3 \\
\hline La Granja & 11 & 446 & 6.041 & 18,2 & 6.012 & 74,2 & & 8,0 \\
\hline El Bosque & 14 & 535 & 7.971 & 17,6 & 8.058 & 66,4 & & 6,5 \\
\hline San Ramón & 7 & 333 & 4.044 & 17,3 & 3.815 & 87,2 & & 9,2 \\
\hline Huechuraba & 6 & 200 & 3.789 & 15,8 & 3.303 & 60,5 & & 7,0 \\
\hline Pudahuel & 17 & 486 & 10.789 & 15,8 & 10.339 & 47,0 & & 6,6 \\
\hline Recoleta & 8 & 367 & 5.720 & 14,0 & 5.653 & 64,9 & & 8,5 \\
\hline Conchalí & 7 & 314 & 5.117 & 13,7 & 5.041 & 62,2 & & 7,6 \\
\hline Renca & 8 & 408 & 6.125 & 13,1 & 4.321 & 69,7 & & 8,8 \\
\hline Independencia & 2 & 119 & 2.130 & 9,4 & 2.198 & 54,1 & & 7,8 \\
\hline Macul & 4 & 154 & 4.249 & 9,4 & 4.318 & 35,7 & & 5,8 \\
\hline Quilicura & 7 & 321 & 7.830 & 8,9 & 6.846 & 46,9 & & 4,9 \\
\hline Lo Barnechea & 3 & 83 & 4.470 & 6,7 & 4.293 & 19,3 & & 5,2 \\
\hline La Reina & 2 & 75 & 4.202 & 4,8 & 4.321 & 17,3 & & 3,4 \\
\hline Las Condes & 4 & 92 & 9.050 & 4,4 & 10.291 & 8,9 & & 0,5 \\
\hline Providencia & 1 & 50 & 2.751 & 3,6 & 3.641 & 13,7 & 10,8 & 0,0 \\
\hline Vitacura & 0 & 11 & 2.855 & 0,0 & 3.210 & 3,4 & & 0,04 \\
\hline
\end{tabular}

* Porcentaje de las raciones alimenticias a escolares del total de la población de cada comuna.

* No se expresa el porcentaje por población escolar por no tener la información por edades simples por comuna.

las comunas con menor proporción de raciones alimenticias.

La comuna de Cerrillos tiene un riesgo de fecundidad 35 veces mayor, en niñas menores de 14 años, en comparación con la comuna de Vitacura (35,6 versus 0,0). El promedio de la fecundidad en 14 años y menos fue 22,2 por 1.000 en las siete primeras comunas y 3,2 en las últimas cuatro. La fecundidad fue 6,9 veces mayor en las comunas donde los escolares reciben mayor proporción de raciones alimenticias.

La fecundidad en adolescentes de 15 a 19 años fue 71,2 por 1.000 en las 7 comunas con mayores proporciones de raciones alimenticias escolares y
10,8 en las 4 comunas con menores raciones. En el primer grupo de comunas, la fecundidad fue 6,6 veces más que en el segundo grupo.

\section{Discusión}

El presente trabajo es la primera información a nivel nacional y total para todas las edades de la fecundidad en menores de 14 años. El número promedio anual de NV registrados de madres de 11 a 14 y de 11 a 15 años, corresponde a 2,7\% y $9,4 \%$, respectivamente, de los $\mathrm{NV}$ de madres adolescentes de 11 a 19 años. Estas bajas propor- 
ciones son importantes por su significado cualitativo social y cultural y por ser un indicador demográfico trazador de otras situaciones sociales de mucho riesgo, como lo fue la mortalidad infantil en décadas pasadas.

No hay muchos estudios acerca del impacto biológico, psíquico y social de la maternidad en los inicios de la pubertad. En Chile, se encontró proporciones de $4 \%$ y $25,7 \%$, de adolescentes de 14 y menos y de 15 años de edad, respectivamente, en un centro especializado en atención de adolescentes al cual fueron referidas de su alto riesgo obstétrico y perinatal o por abusos sexuales crónicos e intrafamiliares ${ }^{11,12}$. El grupo más complejo fue el de 14 años y menos, tanto por las situaciones de extrema irregularidad familiar, discontinuidad de los estudios y serios daños en la salud mental de las niñas-madres. Este grupo concentró los factores de riesgo predictivos materno/perinatal. El sistema de atención médica de alta calidad, preferencial e integral en el control prenatal, parto y recién nacido, aplicado a estas pacientes, permitió prevenir las complicaciones. Sin embargo, en los primeros 5 años de vida de los niños, se presentaron mayores episodios de hospitalizaciones y accidentes ${ }^{11-14}$. Es muy posible que las niñas-madres chilenas tengan riesgos semejantes a lo encontrado en estos cuatro estudios de referencia y que las complicaciones sean de gran magnitud al no tener sistemas especializados de atención, lo que explicaría lo encontrado por Donoso y cols, como se comentó en la introducción ${ }^{4}$.

En 1986, se publicó un estudio de fecundidad adolescente en países industrializados ${ }^{15}$. Se describió que en 1981, en las madres de 14 años y menos, la razón de fecundidad por 1.000 era: Canadá (1,2), Inglaterra y Gales $(0,5)$, Francia $(0,3)$, Holanda $(0,02)$ y Suecia $(0,2)$. El descenso de la fecundidad en esos países, fue por aumento de las tasas de aborto despenalizado en estas edades. Dos décadas después, en 2002, Chile tenía una tasa de 2,0 por 1.000 , es decir 100 y 10 veces más alta que Holanda y Suecia, respectivamente. Las tasas observadas en las comunas de Las Condes $(0,44)$, Providencia $(0,36)$ y Vitacura $(0,0)$, se asemejan a países industrializados. Este fenómeno no puede descartar la hipótesis del mejor acceso a la interrupción clandestina profesionalizada del embarazo ${ }^{16}$. Es necesario recordar que Chile tiene el aborto penalizado por cualquier causa, incluyendo las de causa médica.

Esto reafirma la necesidad de estrategias en la prevención del embarazo inesperado como ha ocurrido en las adultas en Chile. En las menores de 14 años, la educación y los servicios de salud, dirigidos a este grupo, son indispensables, por la mayor mortalidad neonatal, infantil, bajo peso al nacer y de tener un progenitor adolescente ${ }^{4}$.

En un estudio de seguimiento en Chile, se encontró que el riesgo relativo (RR) de abuso sexual en embarazadas menores de 15 años fue de 5,9712. Este aspecto tiene una connotación médico legal que provoca serios problemas a las fiscalías encargadas de estos asuntos con la reforma procesal penal en Chile y, con la reforma de la ley de abusos sexuales que obliga a la denuncia de cualquier embarazo en menores de 14 años, como eventual víctima de abuso sexual ${ }^{17,18}$. Según el presente estudio, sólo por embarazo en estas edades se debe esperar un promedio de 1.050 denuncias anuales. La mayoría de las adolescentes menores de 14 años tienen relaciones sexuales voluntarias y en gran proporción con otro menor de edad ${ }^{11}$. El proceso que sigue la fiscalía, victimiza a estas jóvenes madres y su familia con daños tan serios como una violación. La información aportada por el presente estudio, muestra que es un tema de revisión jurídica, legislativa y que se debería involucrar más al personal profesional de salud y no sólo darles un rol de informantes, como ocurre en la actual ley de abusos sexuales. (Según el último dato registrado, el total de denuncias anuales, por abuso sexual a toda edad, al Instituto Médico Legal en Chile alcanzaba a 999 en 1999).

La gran diferencia de fecundidad entre comunas de la Región Metropolitana, es inaceptable en lo ético, moral y cultural. El nivel de educación seguramente explique las diferencias. Los padres más educados son un estímulo para los proyectos de vida de los hijos y la presencia de una comunicación familiar abierta, franca y en un espacio vital adecuado, desarrolla la autoestima. Estos dos pilares se han demostrado como protectores en estudios de adolescentes de 15 a 19 años ${ }^{19}$.

La reforma en salud de la atención primaria no ha favorecido a la adolescencia. De 42 centros de 
atención para adolescente en los inicios de los 90, en Chile, sólo quedan 3 ó 4 . La reforma, ha privilegiado la creación de centros de atención familiar integral, donde se incluye a los y las adolescentes, que no acuden por causas de salud sexual y reproductiva. La consulta de adolescentes en salud sexual y reproductiva no es apoyada por los padres en los niveles socioeconómicos medios o bajos, como ocurre con los de mayor nivel socioconómico y educacional.

En conclusión, la tendencia al aumento del embarazo en niñas-madres de 14 años y menos en los últimos once años de observación en

\section{REFERENCIAS}

1. TAUCher E, Jofré I. Mortalidad infantil en Chile: El gran descenso. Rev Méd Chil 1997; 10: 1225-35.

2. AHMED O T. UNFPA. Estado de la población mundial 2003. Valorizar 1.000 millones de adolescentes. Editor UNFPA, Impresión Prographics Inc. Anápolis, Maryland, Estados Unidos.

3. The Alan Guttmacher Institute. Into a New World Young women's sexual and reproductive lives. Editor Alan Guttmacher Institute. 2000).

4. Donoso J, Becker L Viшarroel. Embarazo en la adolescente chilena menor de 15 años. Análisis de la última década del siglo XX. Rev Ch Obs Gin 2001; LXVI, 391-6.

5. Luengo X. Características de la adolescencia normal. En: R Molina, Sandoval J, González E. Salud sexual y reproductiva en la adolescencia. Ed. Mediterráneo, 2003.

6. SERNAM, CORSAPS, ICMER, Chile: Situación de la salud y los derechos sexuales y reproductivos. Editores. Icmer/Corsaps, 2003 Santiago.

6. www.ine.cl / anuario de estadísticas vitales 2003, pág. 73, 2005.

7. JARA A. Crecimiento y desarrollo biológico. Maduración endocrino sexual. En: R Molina, Sandoval J, González E. Salud sexual y reproductiva en la adolescencia. Ed. Mediterráneo, 2003.

8. Apter D, Bhtzow TL, Laughlin GA, Yen ScCC. Gonadotropin releasing hormone pulse generator activity during pubertal transition in girls: pulsatil
Chile, es "la punta del iceberg" de un problema serio en salud pública y que se ha transformado en un indicador-trazador, como centinela de otros problemas sociales aún no resueltos. Es importante por el impacto que provoca en la población en edad escolar y en los hijos de estos escolares-padres. La prevención del problema es el desarrollo de la educación sexual y la existencia de servicios de salud para adolescentes en la atención primaria, lo cual implica importantes cambios en las políticas públicas al respecto.

and diurnal patterns of circulating gonadotropins. J Clin Endocrinol Metab 1993; 76: 940.

9. LóPEZ C. Orientaciones técnicas para una modalidad de atención amigable para adolescentes en establecimientos de atención primaria, octubre 2005 www.minsal.cl destacados.

10. www.mineduc.cl/site/servlet/NavigationServlet Ficha Comunal de educación. Datos de educación 2004, 2 de agosto, 2005.

11. GonZÁLEZ E. El varón y la paternidad precoz. En: R Molina, Sandoval J, González E. Salud sexual y reproductiva en la adolescencia. Ed. Mediteráneo, 2003.

12. Mouna R. Abuso sexual en adolescentes: aspectos epidemiológicos. En: Molina R, Sandoval J, González E. Salud sexual y reproductiva en la adolescencia. Ed. Mediterráneo, 2003.

13. Luengo X, González E. Hijos de madres adolescentes. En: Molina R, Sandoval J, González E. Salud sexual y reproductiva en la adolescencia. Ed. Mediterráneo, 2003.

14. Molna R, Luengo X, SandovalJ, Gonzáifz E, Mouna T. Factores de riesgo del embarazo, parto y recién nacido en adolescentes embarazadas. Rev Chil Obstet Ginecol Inf y Adolec 1998; 5: 17-28.

15. The Alan Guttmacher Institute. Teenage Pregnancy in Industrialized Countries. Yale University Press. New Haven and London. Pennsylvania, Michigan, 1986.

16. Molina R, Pereda C, Cumsile F, Martínez O L, Miranda E, Molina T. Prevention of Pregnancy in 
High-Risk Women: Community Intervention in Chile. En: Abortion in the Developing World. Editors Axel Mundigo, Cynthia Indriso, ed, World Helath Organization, Vistar Publications, New Delhi, 1999

17. Ley №19.617. «Modifica el código Penal (artículo 362), el código de procedimiento legal y otros cuerpos legales en materias relativas al delito de violación». Diario oficial de la República de Chile.
Lunes 12 de julio 1999. Normativa Técnica pericial sobre agresiones sexuales. Diario Oficial de la República de Chile, 21 de agosto 1999.

18. Ley № 19.789. «Reforma Procesal Penal»en Chile, Diario Oficial de la República de Chile. Miércoles 30 enero, 2002.

19. ZABIN L Sch. Evaluation Pregnancy prevention Programs in the school Context. Editorial Lexington Books, Massachussets and Canada, 1988. 